Pacific

Journal of

Mathematics

\title{
DEFORMATIONS OF NEARLY KÄHLER STRUCTURES
}

Andrei Moroianu, Paul-Andi NAgy AND UWE SEMmELmann 


\title{
DEFORMATIONS OF NEARLY KÄHLER STRUCTURES
}

\author{
Andrei Moroianu, Paul-Andi Nagy and Uwe Semmelmann
}

\begin{abstract}
We study the space of nearly Kähler structures on compact 6-dimensional manifolds. In particular, we prove that the space of infinitesimal deformations of a strictly nearly Kähler structure (with scalar curvature scal) modulo the group of diffeomorphisms is isomorphic to the space of primitive coclosed $(1,1)$-eigenforms of the Laplace operator for the eigenvalue 2 scal $/ 5$.
\end{abstract}

\section{Introduction}

A nearly Kähler manifold is an almost Hermitian manifold $(M, g, J)$ with the property that $\left(\nabla_{X} J\right) X=0$ for all tangent vectors $X$, where $\nabla$ denotes the LeviCivita connection of $g$. A nearly Kähler manifold is called strictly nearly Kähler if $\left(\nabla_{X} J\right)$ is nonzero for every nonzero tangent vector $X$. Besides Kähler manifolds, there are two main families of examples of compact nearly Kähler manifolds: naturally reductive 3-symmetric spaces, which are classified by A. Gray and J. Wolf [1968]; and twistor spaces over compact quaternion-Kähler manifolds with positive scalar curvature that are endowed with the nonintegrable canonical almost complex structure (see [Nagy 2002]).

A nearly Kähler manifold of dimension 4 is automatically a Kähler surface, and the only known examples of non-Kähler compact nearly Kähler manifolds in dimension 6 are the 3 -symmetric spaces $G_{2} / \mathrm{SU}_{3}, \mathrm{SU}_{3} / S^{1} \times S^{1}, \mathrm{Sp}_{2} / S^{1} \times \mathrm{Sp}_{1}$, and $\mathrm{Sp}_{1} \times \mathrm{Sp}_{1} \times \mathrm{Sp}_{1} / \mathrm{Sp}_{1}$. Moreover, J.-B. Butruille [2005] has recently shown that there are no other homogeneous examples in dimension 6.

On the other hand, using previous results of R. Cleyton and A. Swann on $G$ structures with skew-symmetric intrinsic torsion, Nagy [2002] proved that every compact simply connected nearly Kähler manifold $M$ is isometric to a Riemannian product $M_{1} \times \cdots \times M_{k}$, such that for each $i, M_{i}$ is a nearly Kähler manifold belonging to the following list: Kähler manifolds, naturally reductive 3-symmetric spaces, twistor spaces over compact quaternion-Kähler manifolds with positive scalar curvature, and 6-dimensional nearly Kähler manifolds.

MSC2000: 58E30, 53C10, 53C15.

Keywords: infinitesimal deformations, $\mathrm{SU}_{3}$ structures, nearly Kähler manifolds, Gray manifolds. 
It is thus natural to concentrate on the 6-dimensional case, especially because in this dimension non-Kähler nearly Kähler manifolds have several interesting features: they carry real Killing spinors (and thus are automatically Einstein with positive scalar curvature) and they are defined by a $\mathrm{SU}_{3}$ structure whose intrinsic torsion is skew-symmetric. These manifolds were intensively studied by A. Gray in the 70's, thus motivating the following

Definition. A compact strictly nearly Kähler manifold of dimension 6 is called a Gray manifold.

Our main goal is to study the deformation problem for Gray manifolds. We consider simultaneous deformations of the metric and of the almost complex structure. Indeed M. Verbitsky [2007] proved that on a 6-dimensional almost complex manifold there is up to constant rescaling at most one strictly nearly Kähler metric. Conversely it is well known (see [Baum et al. 1991] or Section 4 below) that on a manifold $\left(M^{6}, g\right)$ that is not locally isometric to the standard sphere, there is at most one compatible almost complex structure $J$ such that $(M, g, J)$ is nearly Kähler.

We start by studying deformations of $\mathrm{SU}_{3}$ structures and then use the characterization of Gray manifolds as $\mathrm{SU}_{3}$ structures satisfying a certain exterior differential system in order to compute the space of infinitesimal deformations of a given Gray structure modulo diffeomorphisms. In particular, we prove that this space is isomorphic to some eigenspace of the Laplace operator acting on 2-forms (see Theorem 4.1 for a precise statement).

\section{Algebraic preliminaries}

Let $V$ denote the standard 6-dimensional $\mathrm{SU}_{3}$ representation space, which comes equipped with the Euclidean product $g \in \operatorname{Sym} V^{*}$, the complex structure $J \in$ $\operatorname{End}(V)$, the fundamental 2-form $\omega(\cdot, \cdot)=g(J \cdot, \cdot) \in \Lambda^{2} V^{*}$, and the complex volume element $\psi^{+}+i \psi^{-} \in \Lambda^{(3,0)} V^{*}$.

These objects satisfy the compatibility relations $\omega \wedge \psi^{ \pm}=0$ and $\psi^{+} \wedge \psi^{-}=$ $(2 / 3) \omega^{3}=4 d v$, where $d v$ denotes the volume form of the metric $g$. It is easy to check that $\psi^{+}$and $\psi^{-}$are related by

$$
\psi^{-}(X, Y, Z):=-\psi^{+}(J X, Y, Z) .
$$

We identify elements of $V$ and $V^{*}$ using the isomorphism induced by $g$. For any orthonormal basis $\left\{e_{i}\right\}$ of $V$ adapted to $J$ (that is, $\left.J\left(e_{2 i-1}\right)=e_{2 i}\right)$ we have

$$
\begin{gathered}
\omega=e^{12}+e^{34}+e^{56}, \\
\psi^{+}=e^{135}-e^{146}-e^{236}-e^{245}, \quad \psi^{-}=e^{136}+e^{145}+e^{235}-e^{246} .
\end{gathered}
$$


The following formulas are straightforward (it is enough to check them for $X=e_{1}$ and use the transitivity of the $\mathrm{SU}_{3}$ action on spheres):

$$
\begin{aligned}
& \left.\psi^{+} \wedge(X\lrcorner \psi^{+}\right)=X \wedge \omega^{2}, \\
& \left.\psi^{+} \wedge(X\lrcorner \psi^{-}\right)=-J X \wedge \omega^{2}, \\
& \left.\psi^{-} \wedge(X\lrcorner \psi^{+}\right)=J X \wedge \omega^{2} \text {, } \\
& \left.\psi^{-} \wedge(X\lrcorner \psi^{-}\right)=X \wedge \omega^{2} \text {. }
\end{aligned}
$$

Let $\Lambda: \Lambda^{p} V \rightarrow \Lambda^{p-2} V$ denote the metric adjoint of the wedge product with $\omega$, that is, $\left.\left.\Lambda=\frac{1}{2} \sum_{i} J e_{i}\right\lrcorner e_{i}\right\lrcorner$. It is easy to check that

$$
\left.\left.\Lambda(X\lrcorner \psi^{ \pm}\right)=0 \quad \text { and } \quad \Lambda\left(X \wedge \psi^{ \pm}\right)=J X\right\lrcorner \psi^{ \pm} \quad \text { for all } X \in V
$$

and

$$
\Lambda(\tau \wedge \omega)=\omega \wedge \Lambda \tau+(3-p) \tau \text { for all } \tau \in \Lambda^{p} V .
$$

We next describe the decomposition into irreducible summands of $\Lambda^{2} V$ and $\Lambda^{3} V$. We use the notation $\Lambda^{(p, q)+(q, p)} V$ for the projection of $\Lambda^{(p, q)} V$ onto the real space $\Lambda^{p+q} V$. Then

$$
\Lambda^{2} V=\left(\Lambda_{0}^{(1,1)} V \oplus \mathbb{R} \omega\right) \oplus \Lambda^{(2,0)+(0,2)} V,
$$

where the first two summands consist of $J$-invariant and the last of $J$-antiinvariant forms. Here $\Lambda_{0}^{(1,1)} V$ is the space of primitive $(1,1)$-forms, that is, the kernel of the contraction map $\Lambda$. The map $\xi \mapsto \xi\lrcorner \psi^{+}$defines an isomorphism of the second summand $\Lambda^{(2,0)+(0,2)} V$ with $V$. For 3-forms we have the irreducible decomposition

$$
\Lambda^{3} V=\left(\Lambda^{1} V \wedge \omega\right) \oplus \Lambda^{(3,0)+(0,3)} V \oplus \Lambda_{0}^{(2,1)+(1,2)} V
$$

The second summand $\Lambda^{(3,0)+(0,3)} V$ is 2-dimensional and spanned by the forms $\psi^{ \pm}$. The third summand $\Lambda_{0}^{(2,1)+(1,2)} V$ is 12-dimensional and can be identified with the space of symmetric endomorphisms of $V$ anticommuting with $J$. Because of the Schur lemma, the map given by taking the wedge product with $\omega$ vanishes on the last two summands.

An endomorphism $A$ of $V$ (not necessarily skew-symmetric) acts on $p$-forms by the formula

$$
\left(A_{\star} u\right)\left(X_{1}, \ldots, X_{p}\right):=-\sum_{i=1}^{p} u\left(X_{1}, \ldots, A\left(X_{i}\right), \ldots, X_{p}\right) .
$$

A more convenient way of writing this action is

$$
\left.A_{\star} u=-\sum_{i=1}^{p} A^{*}\left(e_{i}\right) \wedge e_{i}\right\lrcorner u,
$$


where $A^{*}$ denotes the metric adjoint of $A$. Taking $A=J$, we obtain the form spaces $\Lambda^{(p, q)+(q, p)} V$ as eigenspaces of $\left(J_{\star}\right)^{2}$ for the eigenvalues $-(p-q)^{2}$. For example, $J_{\star} \varphi=0$ for any 2 -form $\varphi \in \Lambda^{(1,1)} V$.

Let $\mathrm{Sym}^{-} V$ denote the space of symmetric endomorphisms anticommuting with $J$. This space is clearly invariant by composition with $J$. The map $S \mapsto S_{\star} \psi^{+}$, with $S \in \mathrm{Sym}^{-} V$, defines an isomorphism of SU(3)-representations

$$
\mathrm{Sym}^{-} V \cong \Lambda_{0}^{(2,1)+(1,2)} V,
$$

showing in particular that $\mathrm{Sym}^{-} V$ is irreducible. Taking (1) into account, we remark that for $S \in \mathrm{Sym}^{-} V$ we have $S_{\star} \psi^{+}=(J S)_{\star} \psi^{-}$. Notice that $\operatorname{tr}(S)=0$ for all $S \in \mathrm{Sym}^{-} V$.

Let $h$ be any skew-symmetric endomorphism anticommuting with $J$. Then the map $h \mapsto g(h \cdot, \cdot)$ identifies the space of skew-symmetric endomorphism anticommuting with $J$ with $\Lambda^{(2,0)+(0,2)} V$. Using the isomorphism $\left.\xi \mapsto \xi\right\lrcorner \psi^{+}$we can state this as

Lemma 2.1. An endomorphism $F$ of $V$ anticommuting with $J$ can be written in a unique way $F=S+\psi_{\xi}^{+}$for some $S \in \mathrm{Sym}^{-} V$ and $\xi \in V$, where $\psi_{\xi}^{+}$denotes the skew-symmetric endomorphism of $V$ defined by $g\left(\psi_{\xi}^{+} \cdot, \cdot\right)=\psi^{+}(\xi, \cdot, \cdot)$.

Corresponding to the decomposition of $\Lambda^{3} V$ given in (6), we have

Lemma 2.2. An exterior 3-form $u \in \Lambda^{3} V$ can be written in a unique way

$$
u=\alpha \wedge \omega+\lambda \psi^{+}+\mu \psi^{-}+S_{\star} \psi^{+},
$$

for some $\alpha \in V, \lambda, \mu \in \mathbb{R}$, and $S \in \mathrm{Sym}^{-} V$. Its contraction with $\omega$ satisfies

$$
\Lambda u=2 \alpha .
$$

Proof. The contraction map $\Lambda$ obviously vanishes on $\Lambda^{(3,0)+(0,3)} V \oplus \Lambda_{0}^{(2,1)+(1,2)} V$, so by (5) we have $\Lambda u=\Lambda(\alpha \wedge \omega)=2 \alpha$.

The space of symmetric endomorphisms commuting with $J$ is identified with $\Lambda^{(1,1)} V$ via the map $h \mapsto \varphi(\cdot, \cdot):=g(h J \cdot, \cdot)$, which in particular maps the identity of $V$ to the fundamental form $\omega$. If $\varphi$ is a $(1,1)$-form with corresponding symmetric endomorphism $h$, then $h=h_{0}+(1 / 6) \operatorname{tr}(h)$ id, where $h_{0}$ denotes the trace-free part of $h$. As a consequence of this formula and Schur's Lemma we find that

$$
h_{\star} \psi^{+}=\frac{1}{6} \operatorname{tr}(h) \mathrm{id}_{\star} \psi^{+}=-\frac{1}{2} \operatorname{tr}(h) \psi^{+}
$$

for all symmetric endomorphisms $h$ commuting with $J$.

In the rest of this section we recall several properties and formulas related to the Hodge $*$-operator, which we will use in later computations. We consider the scalar 
product $\langle\cdot, \cdot\rangle$ on $\Lambda^{k} V$ characterized by the fact that the basis

$$
\left\{e_{i_{1}} \wedge \cdots \wedge e_{i_{k}} \mid 1 \leq i_{1}<\cdots<i_{k} \leq 6\right\}
$$

is orthonormal. With respect to this scalar product, the interior and exterior products are adjoint operators:

$$
\langle X\lrcorner \omega, \tau\rangle=\langle\omega, X \wedge \tau\rangle \quad \text { for all } X \in V, \omega \in \Lambda^{k} V \text {, and } \tau \in \Lambda^{k-1} V .
$$

We define the Hodge star operator $*: \Lambda^{k} V \rightarrow \Lambda^{6-k} V$ by

$$
\omega \wedge * \tau:=\langle\omega, \tau\rangle d v \text { for all } \omega, \tau \in \Lambda^{k} V,
$$

where $d v=1 / 6 \omega^{3}$ denotes the volume form ( $d v=e^{123456}$ in our notations). It is well known that the following relations are satisfied:

$$
* \omega=\frac{1}{2} \omega^{2}, \quad\langle * \omega, * \tau\rangle=\langle\omega, \tau\rangle, \quad *^{2}=(-1)^{k} \quad \text { on } \Lambda^{k} V
$$

From the expression of $\psi^{+}$and $\psi^{-}$in any orthonormal basis $\left\{e_{i}\right\}$ we see that $* \psi^{+}=\psi^{-}$and $* \psi^{-}=-\psi^{+}$. For later use we compute the Hodge operator on $\Lambda_{0}^{(2,1)+(1,2)} V$, too. Let $S \in \operatorname{Sym}^{-} V$ and $\alpha \in \Lambda_{0}^{(2,1)+(1,2)} V$. We have $\alpha \wedge \psi^{-}=0$, whence

$$
\begin{aligned}
\left\langle\alpha, S_{\star} \psi^{+}\right\rangle d v & \left.\left.\stackrel{\text { by (8) }}{=}-\sum_{i}\left\langle\alpha, S\left(e_{i}\right) \wedge e_{i}\right\lrcorner \psi^{+}\right\rangle d v=-\sum_{i}\left\langle e_{i} \wedge S\left(e_{i}\right)\right\lrcorner \alpha, \psi^{+}\right\rangle d v \\
& =\left(S_{\star} \alpha\right) \wedge * \psi^{+}=\left(S_{\star} \alpha\right) \wedge \psi^{-}=S_{\star}\left(\alpha \wedge \psi^{-}\right)-\alpha \wedge\left(S_{\star} \psi^{-}\right) \\
& =-\alpha \wedge\left(S_{\star} \psi^{-}\right)=\left\langle\alpha, *\left(S_{\star} \psi^{-}\right)\right\rangle d v,
\end{aligned}
$$

where we used that $*^{2}=-1$ on 3 -forms to get the last equality. This shows that

$$
*\left(S_{\star} \psi^{-}\right)=S_{\star} \psi^{+} \quad \text { and } \quad *\left(S_{\star} \psi^{+}\right)=-S_{\star} \psi^{-} .
$$

There are two other formulas which we will use later. Let $\varphi_{0}$ be a primitive $(1,1)$ form, let $\xi \in V$, and let $\alpha \in \Lambda^{p} V$ then

$$
\left.*\left(\varphi_{0} \wedge \omega\right)=-\varphi_{0} \quad \text { and } \quad *(\xi \wedge \alpha)=(-1)^{p} \xi\right\lrcorner * \alpha .
$$

\section{Deformations of $\mathrm{SU}_{3}$ structures}

Let $M$ be a smooth 6-dimensional manifold.

Definition. An $\mathrm{SU}_{3}$ structure on $M$ is a reduction of the frame bundle of $M$ to $\mathrm{SU}_{3}$. It consists of a 5-tuple $\left(g, J, \omega, \psi^{+}, \psi^{-}\right)$, where $g$ is a Riemannian metric, $J$ is a compatible almost complex structure, $\omega$ is the corresponding fundamental 2-form $\omega(\cdot, \cdot)=g(J \cdot, \cdot)$, and $\psi^{+}+i \psi^{-}$is a complex volume form of type $(3,0)$. 
If $M$ carries an $\mathrm{SU}_{3}$ structure, each tangent space $T_{x} M$ has an $\mathrm{SU}_{3}$ representation isomorphic to the standard one, and so all algebraic results of the previous section transfer verbatim to global results on $M$. In what remains, we will usually identify tangent vectors and 1-forms on $M$ using the isomorphism induced by the metric $g$.

Let $\left(g_{t}, J_{t}, \omega_{t}, \psi_{t}^{+}, \psi_{t}^{-}\right)$be a smooth family of $\mathrm{SU}_{3}$ structures on $M$. We omit the index $t$ when the above tensors are evaluated at $t=0$, and we use the dot to denote the derivative at $t=0$ in the direction of $t$.

We start with the study of the 1-jet at $t=0$ of the family of $\mathrm{U}_{3}$ structures $\left(g_{t}, J_{t}, \omega_{t}\right)$.

Lemma 3.1. There exist a vector field $\xi$, a section $S$ of $\mathrm{Sym}^{-} M$ and a section $h$ of $\mathrm{Sym}^{+} M$ (that is, symmetric endomorphism commuting with $J$ ) such that

$$
\begin{aligned}
& \dot{g}=g((h+S) \cdot, \cdot), \\
& \dot{J}=J S+\psi_{\xi}^{+}, \\
& \dot{\omega}=\varphi+\xi\lrcorner \psi^{+},
\end{aligned}
$$

where $\varphi$ is the $(1,1)$-form defined by $\varphi(\cdot, \cdot)=g(h J \cdot, \cdot)$.

Proof. Let us write $g_{t}(\cdot, \cdot)=g\left(f_{t} \cdot, \cdot\right)$, so that $\dot{g}(\cdot, \cdot)=g(\dot{f} \cdot, \cdot)$. We then denote by $h:=(1 / 2)(\dot{f}-J \dot{f} J)$ and $S:=(1 / 2)(\dot{f}+J \dot{f} J)$ the $J$-invariant and $J$-antiinvariant parts of $\dot{f}$, which are clearly $g$-symmetric endomorphisms. This proves the first relation, which actually holds for deformations of almost Hermitian structures in all dimensions.

Since $J_{t}^{2}=-\mathrm{id}_{T M}$, we see that $\dot{J}$ anticommutes with $J$. Lemma 2.1 then shows that $\dot{J}=\tilde{S}+\psi_{\xi}^{+}$for some section $\tilde{S}$ of $\operatorname{Sym}^{-} M$ and some vector field $\xi$. Differentiating the relation $g_{t}\left(J_{t} X, Y\right)+g_{t}\left(X, J_{t} Y\right)=0$ yields

$$
0=\dot{g}(J X, Y)+\dot{g}(X, J Y)+g(\dot{J} X, Y)+g(X, \dot{J} Y)=2 g(S J X, Y)+2 g(\tilde{S} X, Y),
$$

thus proving $\tilde{S}=-S J=J S$. The last formula follows directly from (14) and (15):

$$
\begin{aligned}
\dot{\omega}(X, Y) & =\dot{g}(J X, Y)+g(\dot{J} X, Y)=g((h+S) J X, Y)+g\left(\left(J S+\psi_{\xi}^{+}\right) X, Y\right) \\
& \left.=g(h J X, Y)+(\xi\lrcorner \psi^{+}\right)(X, Y) .
\end{aligned}
$$

This result actually says that the tangent space to the set of all $\mathrm{U}_{3}$ structures on $M$ at $(g, J, \omega)$ is parametrized by a section $(\xi, S, \varphi)$ of the bundle $T M \oplus \operatorname{Sym}^{-} M \oplus$ $\Lambda^{(1,1)} M$. We now go forward and describe the 1 -jet of a family of $\mathrm{SU}_{3}$ structures. Since the reduction from a $\mathrm{U}_{3}$ structure to a $\mathrm{SU}_{3}$ structure is given by a section in some $S^{1}$-bundle, it is not very surprising that the extra freedom in the tangent space is measured by a real function ( $\mu$ in the notation below): 
Lemma 3.2. The derivatives at $t=0$ of $\psi_{t}^{+}$and $\psi_{t}^{-}$are given by

$$
\begin{aligned}
& \dot{\psi}^{+}=-\xi \wedge \omega+\lambda \psi^{+}+\mu \psi^{-}-\frac{1}{2} S_{\star} \psi^{+}, \\
& \dot{\psi}^{-}=-J \xi \wedge \omega-\mu \psi^{+}+\lambda \psi^{-}-\frac{1}{2} S_{\star} \psi^{-},
\end{aligned}
$$

where $\lambda=(1 / 4) \operatorname{tr}(h)$ and $\mu$ is some smooth function on $M$.

Proof. By Lemma 2.2, we can write

$$
\dot{\psi}^{+}=\alpha \wedge \omega+\lambda \psi^{+}+\mu \psi^{-}+Q_{\star} \psi^{+},
$$

for some functions $\lambda$ and $\mu$, a 1-form $\alpha$, and some section $Q$ of $\operatorname{Sym}^{-} M$.

The fact that $\psi_{t}^{+}$defines - in addition to the $\mathrm{U}_{3}$ structure $\left(g_{t}, J_{t}\right)-\mathrm{a} \mathrm{SU}_{3}$ structure is characterized by the two equations

$$
g_{t}\left(\psi_{t}^{+}, \psi_{t}^{+}\right)=4 \quad \text { and } \quad \psi_{t}^{+}\left(J_{t} X, Y, Z\right)=\psi_{t}^{+}\left(X, J_{t} Y, Z\right) .
$$

We consider the symmetric endomorphism $f_{t}$ introduced above, which corresponds to $g_{t}$ in the ground metric $g$. Since the identity acts on 3 -forms by -3 id, the first part of (20) reads $g\left(f_{t \star} \psi_{t}^{+}, \psi_{t}^{+}\right)=-12$. Differentiating this at $t=0$ and using the fact that $\psi^{+}$and $S_{\star} \psi^{+}$live in orthogonal components of $\Lambda^{3} M$, we obtain

$$
\begin{aligned}
& 0=g\left(\dot{f}_{\star} \psi^{+}, \psi^{+}\right)-6 g\left(\dot{\psi}^{+}, \psi^{+}\right)=g\left((h+S)_{\star} \psi^{+}, \psi^{+}\right)-24 \lambda \\
& \stackrel{\text { by }(10)}{=} 6 \operatorname{tr}(h)-24 \lambda .
\end{aligned}
$$

This determines the function $\lambda$. We next differentiate the identity $\psi_{t}^{+} \wedge \omega_{t}=0$ at $t=0$. Since the wedge product with $\omega$ vanishes on $\psi^{+}$and $\psi^{-}$and on $Q_{\star} \psi^{+} \in$ $\Lambda_{0}^{(2,1)+(1,2)} M$, we get

$$
\left.0=\dot{\psi}^{+} \wedge \omega+\psi^{+} \wedge \dot{\omega}=\alpha \wedge \omega^{2}+\psi^{+} \wedge(\varphi+\xi\lrcorner \psi^{+}\right) \stackrel{\text { by }(2)}{=}(\alpha+\xi) \wedge \omega^{2},
$$

showing that $\alpha=-\xi$. Finally, we differentiate the second part of (20) at $t=0$ :

$$
\dot{\psi}^{+}(J X, Y, Z)+\psi^{+}(\dot{J} X, Y, Z)=\dot{\psi}^{+}(X, J Y, Z)+\psi^{+}(X, \dot{J} Y, Z) .
$$

Using (15) and (19), this is equivalent to the expression

$$
-(\xi \wedge \omega)(J X, Y, Z)+\left(Q_{\star} \psi^{+}\right)(J X, Y, Z)+\psi^{+}(J S X, Y, Z)+\psi^{+}\left(\psi_{\xi}^{+} X, Y, Z\right)
$$

being skew-symmetric in $X$ and $Y$. It is easy to check that

$$
-(\xi \wedge \omega)(J X, Y, Z)+\psi^{+}\left(\psi_{\xi}^{+} X, Y, Z\right)=(J \xi \wedge \omega)(X, Y, Z) ;
$$

therefore the above condition reduces to

$$
\left(Q_{\star} \psi^{+}\right)(J X, Y, Z)+\psi^{+}(J S X, Y, Z)=\left(Q_{\star} \psi^{+}\right)(X, J Y, Z)+\psi^{+}(X, J S Y, Z) \text {. }
$$


Using (7), this last relation becomes

$$
\psi^{+}((2 Q+S) J X, Y, Z)=\psi^{+}(X,(2 Q+S) J Y, Z) \text { for all } X, Y, Z \in T M \text {. }
$$

The set of all elements of the form $2 Q+S$ satisfying this relation is a $\mathrm{SU}_{3}$ invariant subspace of $\mathrm{Sym}^{-} M$. But $\mathrm{Sym}^{-} M$ is irreducible, and not every element of $\mathrm{Sym}^{-} M$ satisfies (21) (to see this, just pick any element in $\mathrm{Sym}^{-} M$ and check directly). This shows that $2 Q+S=0$.

Finally, (18) is a straightforward consequence of (17). We simply differentiate the formula $J_{t \star} \psi_{t}^{+}=3 \psi_{t}^{-}$(obtained from (1)) at $t=0$ and compute.

Summarizing, we have shown that the tangent space to the set of all $\mathrm{SU}_{3}$ structures on $M$ at $\left(g, J, \omega, \psi^{+}, \psi^{-}\right)$is parametrized by a section $(\xi, S, \varphi, \mu)$ of the vector bundle $T M \oplus \operatorname{Sym}^{-} M \oplus \Lambda^{(1,1)} M \oplus \mathbb{R} M$, where $\mathbb{R} M$ is the trivial line bundle over $M$.

Let $\alpha: \Lambda^{2} M \rightarrow T M$ denote the metric adjoint of the linear map

$$
X \in T M \mapsto X\lrcorner \psi^{+} \in \Lambda^{2} M .
$$

A simple check shows that

$$
\left.\left.\alpha(X\lrcorner \psi^{+}\right)=2 X, \quad \alpha(X\lrcorner \psi^{-}\right)=-2 J X, \quad \alpha(\tau)=0 \quad \text { for all } \tau \in \Lambda^{(1,1)} M .
$$

Using the map $\alpha$, we derive a useful relation between the components $\dot{\psi}^{+}$and $\dot{\omega}$ of any infinitesimal $\mathrm{SU}_{3}$ deformation:

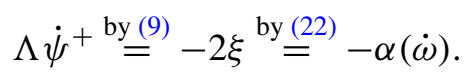

\section{Deformations of Gray manifolds}

Definition. A Gray structure on a 6-dimensional manifold $M$ is a $\mathrm{SU}_{3}$ structure $G:=\left(g, J, \omega, \psi^{+}, \psi^{-}\right)$that satisfies the exterior differential system

$$
\begin{aligned}
& d \omega=3 \psi^{+}, \\
& d \psi^{-}=-2 \omega \wedge \omega
\end{aligned}
$$

A Gray manifold is a compact manifold endowed with a Gray structure.

Since $\mathrm{SU}_{3} \subset \mathrm{Spin}_{6}$, every Gray manifold is automatically spin. It follows from the work of Reyes-Carrión [1993] that a Gray manifold is a strictly nearly Kähler 6-dimensional compact manifold with scalar curvature scal $=30$. We refer to [Gray 1976] for an introduction to nearly Kähler geometry. We will use later on the relations $\left.\nabla_{X} \omega=X\right\lrcorner \psi^{+}$and $\nabla_{X} \psi^{+}=-X \wedge \omega$, which show that $\nabla \omega$ and $\nabla \psi^{+}$are $\mathrm{SU}_{3}$-invariant tensor fields on $M$. Moreover the second equation immediately implies that $\psi^{+}$and $\omega$ are both eigenforms of the Laplace operator for the eigenvalue 12 . 
Note let $M$ be a compact 6-dimensional manifold with some Gray structure $G$ on it. We denote by $\mathfrak{M}$ the connected component of $G$ in the space of Gray structures on $M$. Let $\mathfrak{D}$ be the group of diffeomorphisms of $M$ isotopic to the identity. This group acts on $\mathfrak{M}$ by pull-back and the orbits of this action form the moduli space of deformations of $G$.

The 1-jet of a curve of Gray structures $\left(g_{t}, J_{t}, \omega_{t}, \psi_{t}^{+}, \psi_{t}^{-}\right)$at $G$ becomes at the infinitesimal level a tuple $\gamma:=\left(\dot{g}, \dot{J}, \dot{\omega}, \dot{\psi}^{+}, \dot{\psi}^{-}\right)$determined by a section $(\xi, S, \varphi, \mu)$ of the bundle $T M \oplus \operatorname{Sym}^{-} M \oplus \Lambda^{(1,1)} M \oplus \mathbb{R} M$ via (15)-(18), which satisfies the linearized system of (24), that is,

$$
\begin{aligned}
& d \dot{\omega}=3 \dot{\psi}^{+}, \\
& d \dot{\psi}^{-}=-4 \dot{\omega} \wedge \omega .
\end{aligned}
$$

The space of all tuples $\gamma$ is called the virtual tangent space of $\mathfrak{M}$ at $G$ and is denoted by $T_{G} \mathfrak{M}$. The Lie algebra $\chi(M)$ of $\mathfrak{D}$ maps to $T_{G} \mathfrak{M}$ by $X \mapsto \mathscr{L}_{X} G$. Its image, denoted by $\chi^{G}(M)$, is a vector space isomorphic to $\chi(M) / \mathfrak{K}(g)$, where $\mathfrak{K}(g)$ denotes the set of Killing vector fields on $M$ with respect to $g$. The space of infinitesimal Gray deformations of $G$ is, by definition, the vector space quotient $T_{G} \mathfrak{M} / \chi^{G}(M)$.

The main purpose of this section is to precisely describe this space.

Theorem 4.1. Let $G:=\left(g, J, \omega, \psi^{+}, \psi^{-}\right)$be a Gray structure on a manifold $M$ such that $(M, g)$ is not the round sphere $S^{6}$. Then the space of infinitesimal deformations of $G$ is isomorphic to the eigenspace for the eigenvalue 12 of the restriction of the Laplace operator $\Delta$ to the space of coclosed primitive $(1,1)$-forms $\Lambda_{0}^{(1,1)} M$.

Proof. A simple but very useful remark is that (except on the round sphere $S^{6}$ ), a Gray structure is completely determined by its underlying Riemannian metric. The reason is that the metric defines a unique line of Killing spinors with positive Killing constant, which, in turn, defines the almost complex structure, and, together with the exterior derivative of the Kähler form, one recovers the whole $\mathrm{SU}_{3}$ structure.

We claim that the dependence of the Gray structure on the metric is smooth. Let $\Sigma M$ denote the spin bundle of $M$. By [Baum et al. 1991, p. 137], there is (up to rescalings) exactly one Killing spinor $\Psi$ with Killing constant $1 / 2$, which is obtained as a section of $\Sigma M$, that is parallel with respect to the modified connection $\tilde{\nabla}_{X}:=\nabla_{X}-(1 / 2) X$. (here "." denotes the Clifford product). Since $\tilde{\nabla}$ depends smoothly on $g$, so does $\Psi$. The almost complex structure $J$ is then defined by the equation $J X \cdot \Psi=i X \cdot \bar{\Psi}$ (see [Baum et al. 1991, p. 136]), and so $J$ depends smoothly on $\Psi$. Finally, since the Kähler form $\omega(\cdot, \cdot)=g(J \cdot, \cdot)$ depends smoothly on $g$, so does its exterior derivative $d \omega=\psi^{+}$. 
By the Ebin slice theorem [Berger and Ebin 1969], each infinitesimal deformation of $G$ has a unique representative $\gamma=\left(\dot{g}, \dot{J}, \dot{\omega}, \dot{\psi}^{+}, \dot{\psi}^{-}\right) \in T_{G} \mathfrak{M}$ such that

$$
\delta \dot{g}=0 \quad \text { and } \quad \operatorname{tr}_{g} \dot{g}=0 .
$$

Let $(\xi, S, \varphi, \mu)$ be the section of the bundle $T M \oplus \operatorname{Sym}^{-} M \oplus \Lambda^{(1,1)} M \oplus \mathbb{R} M$ determined by $\gamma$ via the equations (15)-(18). We have to interpret the system of (25) and (26) in terms of $(\xi, S, \varphi, \mu)$.

We start by taking the exterior product with $\psi^{+}$in the first equation of (25) and use (17) to get $d \dot{\omega} \wedge \psi^{+}=3 \dot{\psi}^{+} \wedge \psi^{+}=3 \mu \psi^{-} \wedge \psi^{+}=-12 \mu d v$. On the other hand, using (16) and taking (3) into account yields $\left.d \dot{\omega} \wedge \psi^{+}=d(\varphi+\xi\lrcorner \psi^{+}\right) \wedge \psi^{+}=$ $\left.d\left((\varphi+\xi\lrcorner \psi^{+}\right) \wedge \psi^{+}\right)=d\left(\xi \wedge \omega^{2}\right)$, whence

$$
-12 \mu d v=d\left(\xi \wedge \omega^{2}\right) .
$$

We apply the contraction $\Lambda$ to the first equation of (25) and use (9), (16), and (17):

$$
\left.-6 \xi=3 \Lambda \dot{\psi}^{+}=\Lambda d \dot{\omega}=\Lambda d \varphi+\Lambda d(\xi\lrcorner \psi^{+}\right) .
$$

To compute the last term, we apply the general formula (23) to the particular deformation of the $\mathrm{SU}_{3}$ structure defined by the flow of $\xi: \Lambda\left(\mathscr{L}_{\xi} \psi^{+}\right)=-\alpha\left(\mathscr{L}_{\xi} \omega\right)$. Since $d \omega=3 \psi^{+}$and $d \psi^{+}=0$, we get

$$
\begin{aligned}
\left.\Lambda d(\xi\lrcorner \psi^{+}\right)=\Lambda\left(\mathscr{L}_{\xi} \psi^{+}\right) & =-\alpha\left(\mathscr{L}_{\xi} \omega\right) \\
& =-\alpha(d(\xi\lrcorner \omega))-\alpha(\xi\lrcorner d \omega) \stackrel{\text { by }(22)}{=}-\alpha(d J \xi)-6 \xi,
\end{aligned}
$$

which, together with (28), yields

$$
\Lambda(d \varphi)=\alpha(d J \xi) .
$$

We now examine the second equation of the system (25). From (18) we get

$$
d \dot{\psi}^{-}=-d J \xi \wedge \omega+3 J \xi \wedge \psi^{+}-d \mu \wedge \psi^{+}+d \lambda \wedge \psi^{-}-2 \lambda \omega^{2}-\frac{1}{2} d\left(S_{\star} \psi^{-}\right) .
$$

We apply the contraction $\Lambda$ to this formula and use the second equation of (25) together with (4) and (5):

$$
\begin{aligned}
-4 \dot{\omega}= & \Lambda(-4 \dot{\omega} \wedge \omega)+4(\Lambda \dot{\omega}) \omega=\Lambda d \dot{\psi}^{-}+4(\Lambda \dot{\omega}) \omega \\
=- & \left.\left.d J \xi-\Lambda(d J \xi) \omega-3 \xi\lrcorner \psi^{+}-J d \mu\right\lrcorner \psi^{+}+J d \lambda\right\lrcorner \psi^{-} \\
& \quad-8 \lambda \omega-\frac{1}{2} \Lambda d\left(S_{\star} \psi^{-}\right)+4(\Lambda \dot{\omega}) \omega .
\end{aligned}
$$

Applying $\alpha$ to this equality and using (1), (16) and (23) yields

$$
-8 \xi=-\alpha(d J \xi)-6 \xi-2 J d \mu+2 d \lambda-\frac{1}{2} \alpha \Lambda d\left(S_{\star} \psi^{-}\right) .
$$


From (29) we then get

$$
\xi=J d \mu+\frac{1}{2} \Lambda d \varphi-d \lambda+\frac{1}{4} \alpha \Lambda d\left(S_{\star} \psi^{-}\right) .
$$

Lemma 4.2. Let $\left(g, J, \omega, \psi^{+}, \psi^{-}\right)$be a Gray structure on a manifold $M$. For every section $S$ of $\mathrm{Sym}^{-} M$ and $(1,1)$-form $\varphi$, the following relations hold:

$$
\begin{aligned}
\Lambda d \varphi & =\delta h+2 d \lambda \\
\delta h & =-J \delta \varphi \\
\delta\left(S_{\star} \psi^{+}\right) & \left.=-\Lambda d\left(S_{\star} \psi^{-}\right)-2 \delta S\right\lrcorner \psi^{+} \\
\alpha \Lambda d\left(S_{\star} \psi^{-}\right) & =-2 \delta S \\
\Lambda \delta\left(S_{\star} \psi^{+}\right) & =0
\end{aligned}
$$

where $h$ is the endomorphism defined by $\varphi(\cdot, \cdot)=g(h J \cdot, \cdot)$ and $\lambda=(1 / 4) \operatorname{tr}(h)=$ $(1 / 2) \Lambda \varphi$. In the above formulas, $\delta$ stands for the usual codifferential when applied to an exterior form but stands for the divergence operator (see [Besse 1987, 1.59]) when acting on symmetric tensors.

Since the proof is rather technical, we postpone it to the end of this section.

Using (14), (32), (33) and (35), the relation (31) becomes

$$
\xi=J d \mu+\frac{1}{2} \delta h-\frac{1}{2} \delta S=J d \mu-J \delta \varphi-\frac{1}{2} \delta \dot{g} .
$$

From (26) and (37) we obtain

$$
\xi=J d \mu-J \delta \varphi .
$$

On the other hand, (38) shows that $\mu$ is an eigenfunction of $\Delta$ with eigenvalue 6:

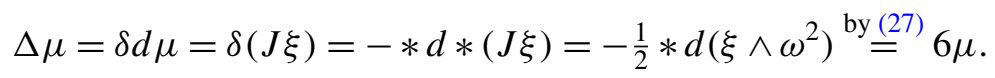

Now, the Obata theorem (see [Obata 1962, Theorem 3]) says that on a compact $n$-dimensional Einstein manifold of positive scalar curvature scal, every eigenvalue of the Laplace operator is greater than or equal to scal/ $(n-1)$, and equality can only occur on the standard sphere. Since $(M, g)$ is not isometric to the standard sphere and is Einstein with scalar curvature scal $=30$, the Obata theorem thus implies that $\mu=0$. Since $\lambda=(1 / 4) \operatorname{tr}(h)=(1 / 4) \operatorname{tr}(\dot{g})$, the second part of (26) also shows that $\lambda=0$. Taking (26), (33), (34), and (38) into account, Equation (30) now becomes

$$
\begin{aligned}
-4 \varphi-4 \xi\lrcorner \psi^{+} & =-4 \dot{\omega}=-d J \xi-\Lambda(d J \xi) \omega-3 \xi\lrcorner \psi^{+}-\frac{1}{2} \Lambda d\left(S_{\star} \psi^{-}\right) \\
& \left.=-d J \xi-\Lambda(d J \xi) \omega-3 \xi\lrcorner \psi^{+}+\frac{1}{2} \delta\left(S_{\star} \psi^{+}\right)-\xi\right\lrcorner \psi^{+},
\end{aligned}
$$

whence

$$
d J \xi=-\Lambda(d J \xi) \omega+4 \varphi+\frac{1}{2} \delta\left(S_{\star} \psi^{+}\right) .
$$


Applying $\Lambda$ to this relation and using (36) yields $\Lambda(d J \xi)=3 \Lambda(d J \xi)$, and so $d J \xi=4 \varphi+\frac{1}{2} \delta\left(S_{\star} \psi^{+}\right)$. By (38) we have $J \xi=\delta \varphi$, and thus $\Delta(J \xi)=\delta d J \xi=$ $4 \delta \varphi=4 J \xi$, that is, $J \xi$ is an eigenform of the Laplace operator with eigenvalue 4 . On the other hand, the Bochner formula on 1-forms,

$$
\Delta=\nabla^{*} \nabla+\operatorname{Ric}=\nabla^{*} \nabla+5 \mathrm{id}
$$

shows, by integration over $M$, that 4 cannot be an eigenvalue of $\Delta$; so $\xi$ must vanish identically. From Lemmas 3.1 and 3.2, we get

$$
\dot{\psi}^{+}=-\frac{1}{2} S_{\star} \psi^{+}, \quad \dot{\psi}^{-}=-\frac{1}{2} S_{\star} \psi^{-}, \quad \dot{\omega}=\varphi \in \Omega_{0}^{(1,1)} M .
$$

Plugging these equations into (25) yields $d \varphi=-\frac{3}{2} S_{\star} \psi^{+}$and

$$
\begin{array}{r}
\delta\left(S_{\star} \psi^{+}\right)=-* d *\left(S_{\star} \psi^{+}\right) \stackrel{\text { by }}{=} * d\left(S_{\star} \psi^{-}\right)=-2 * d \dot{\psi}^{-} \\
\stackrel{\text { by (25) }}{=} 8 *(\dot{\omega} \wedge \omega) \stackrel{\text { by }(13)}{=}-8 \varphi .
\end{array}
$$

Thus $\varphi$ is a coclosed eigenform of the Laplace operator for the eigenvalue 12 .

Conversely let us assume that $\varphi \in \Omega_{0}^{(1,1)} M$ is coclosed and satisfies $\Delta \varphi=12 \varphi$. We have to show that $\varphi$ defines an infinitesimal deformation of the given Gray structure. The main point is to remark that $d \varphi$ is a form in $\Omega_{0}^{(2,1)+(1,2)} M$.

Lemma 4.3. If $\varphi$ is a coclosed form in $\Omega_{0}^{(1,1)} M$, then $d \varphi \in \Omega_{0}^{(2,1)+(1,2)} M$.

Proof. Using Lemma 2.2, this amounts to checking that $d \varphi$ satisfies the system

$$
\begin{aligned}
d \varphi \wedge \psi^{+} & =0, \\
d \varphi \wedge \psi^{-} & =0, \\
\langle d \varphi, X \wedge \omega\rangle & =0 \quad \text { for all } X \in T M .
\end{aligned}
$$

Each of these equations follows easily:

$$
\begin{aligned}
& d \varphi \wedge \psi^{+}=d\left(\varphi \wedge \psi^{+}\right)=0, \\
& d \varphi \wedge \psi^{-}=d\left(\varphi \wedge \psi^{-}\right)-\varphi \wedge d \psi^{-} \stackrel{\text { by } \stackrel{(24)}{=}}{=} 2 \varphi \wedge \omega^{2} \stackrel{\text { by } \stackrel{(11)}{=}}{=} 4\langle\varphi, \omega\rangle=0,
\end{aligned}
$$

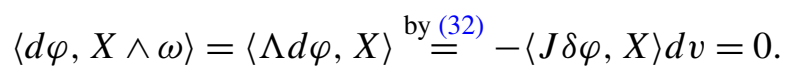

Consequently, there is a unique section $S$ of $\operatorname{Sym}^{-} M$ with $d \varphi=-(3 / 2) S_{\star} \psi^{+}$. Taking $\xi=0, \lambda=0$, and $\mu=0$, the equations (15)-(18) define an infinitesimal $\mathrm{SU}_{3}$ deformation by $\dot{\omega}:=\varphi, \dot{\psi}^{+}:=-(1 / 2) S_{\star} \psi^{+}$, and $\dot{\psi}^{-}:=-(1 / 2) S_{\star} \psi^{-}$. It remains to show that $\left(\dot{\omega}, \dot{\psi}^{+}, \dot{\psi}^{-}\right)$satisfy the linearized system (25). The first equation is clear by definition, and the second is equivalent to $d\left(S_{\star} \psi^{-}\right)=8 \varphi \wedge \omega$. Using again (12) and (13), this last equation is equivalent to $\delta\left(S_{\star} \psi^{+}\right)=-8 \varphi$, which follows directly from the hypothesis on $\varphi$ and the definition of $S$. 
Proof of Lemma 4.2. By the isomorphism $\xi \mapsto \xi\lrcorner \psi^{+}$, we have $\nabla_{X} J=\psi_{X}^{+} \in$ $\Lambda^{(0,2)+(2,0)} M$, and so $\left\langle\varphi, \nabla_{X} J\right\rangle=0$ for all vectors $X$. Identifying $\varphi$ with the corresponding skew-symmetric endomorphism of $T M$, we compute in a local orthonormal basis $\left\{e_{i}\right\}$ parallel at some point:

$$
\begin{aligned}
\Lambda d \varphi & \left.\left.\left.\left.=\frac{1}{2} J e_{i}\right\lrcorner e_{i}\right\lrcorner\left(e_{k} \wedge \nabla_{e_{k}} \varphi\right)=\frac{1}{2} J e_{i}\right\lrcorner \nabla_{e_{i}} \varphi-\frac{1}{2} J e_{i}\right\lrcorner\left(e_{k} \wedge\left(\nabla_{e_{k}} \varphi\right) e_{i}\right) \\
& =\frac{1}{2}\left(\nabla_{e_{i}} \varphi\right) J e_{i}-\frac{1}{2}\left(\nabla_{J e_{i}} \varphi\right) e_{i}+\frac{1}{2} e_{k}\left(\nabla_{e_{k}} \varphi\right)\left(e_{i}, J e_{i}\right) \\
& =\left(\nabla_{e_{i}} \varphi\right) J e_{i}+d\langle\varphi, \omega\rangle-e_{k} \varphi\left(e_{i},\left(\nabla_{e_{k}} J\right) e_{i}\right) \\
& =\left(\nabla_{e_{i}}(\varphi J)\right) e_{i}-\varphi\left(\left(\nabla_{e_{i}} J\right) e_{i}\right)+2 d \lambda-2 e_{k}\left\langle\varphi, \nabla_{e_{k}} J\right\rangle \\
& =-\left(\nabla_{e_{i}} h\right)\left(e_{i}\right)+2 d \lambda=\delta h+2 d \lambda .
\end{aligned}
$$

This proves (32). To prove the second relation, we notice that

$$
g\left(\left(\nabla_{e_{i}} J\right)\left(\varphi e_{i}\right), X\right)=\psi^{+}\left(e_{i}, \varphi e_{i}, X\right)=2\left\langle\psi_{X}^{+}, \varphi\right\rangle=0,
$$

and so

$$
\delta h=-\left(\nabla_{e_{i}} h\right) e_{i}=\left(\nabla_{e_{i}}(J \varphi)\right) e_{i}=-J \delta \varphi+\left(\nabla_{e_{i}} J\right) \varphi e_{i}=-J \delta \varphi .
$$

We will now use several times (and signal this by a star above the equality sign in the calculations below) the fact that any $\mathrm{SU}_{3}$-equivariant map $\mathrm{Sym}^{-} M \rightarrow \Lambda^{2} M$ is automatically zero by the Schur lemma. For every 3 -form $\tau$ we can write

$$
\begin{aligned}
\Lambda d(\tau) & \left.\left.\left.\left.\left.=\frac{1}{2} J e_{i}\right\lrcorner e_{i}\right\lrcorner\left(e_{k} \wedge \nabla_{e_{k}} \tau\right)=e_{k} \wedge\left(\frac{1}{2} J e_{i}\right\lrcorner e_{i}\right\lrcorner \nabla_{e_{k}} \tau\right)+J e_{k}\right\lrcorner \nabla_{e_{k}} \tau \\
& \left.\left.\left.=d \Lambda \tau-\frac{1}{2} e_{k} \wedge\left(\nabla_{e_{k}} J\right) e_{i}\right\lrcorner e_{i}\right\lrcorner \tau+J e_{k}\right\lrcorner \nabla_{e_{k}} \tau .
\end{aligned}
$$

In particular, for $\tau=S_{\star} \psi^{+}$we have $\Lambda \tau=0$ and $\left.\left.e_{k} \wedge\left(\nabla_{e_{k}} J\right) e_{i}\right\lrcorner e_{i}\right\lrcorner \tau=0$, and so from (8) and the remark above we get

$$
\begin{aligned}
\Lambda d\left(S_{\star} \psi^{-}\right) & \left.\left.\left.=J e_{k}\right\lrcorner \nabla_{e_{k}}\left(S_{\star} \psi^{-}\right)=J e_{k}\right\lrcorner\left(\left(\nabla_{e_{k}} S\right)_{\star} \psi^{-}\right)+J e_{k}\right\lrcorner\left(S_{\star}\left(\nabla_{e_{k}} \psi^{-}\right)\right) \\
& \left.\left.\stackrel{*}{=}-J e_{k}\right\lrcorner\left(\left(\nabla_{e_{k}} S\right) e_{j} \wedge e_{j}\right\lrcorner \psi^{-}\right) \\
& \left.\left.\left.=-\left\langle\left(\nabla_{e_{k}} S\right) e_{j}, J e_{k}\right\rangle e_{j}\right\lrcorner \psi^{-}+\left(\nabla_{e_{k}} S\right) e_{j} \wedge\left(J e_{k}\right\lrcorner e_{j}\right\lrcorner \psi^{-}\right) \\
& \left.\left.\left.\stackrel{*}{=}\left\langle\left(\nabla_{e_{k}} S\right) J e_{j}, e_{k}\right\rangle J e_{j}\right\lrcorner \psi^{+}+\left(\nabla_{e_{k}} S\right) e_{j} \wedge\left(J e_{k}\right\lrcorner e_{j}\right\lrcorner \psi^{-}\right) \\
& \left.\left.=-\delta S\lrcorner \psi^{+}+\left(\nabla_{e_{k}} S\right) e_{j} \wedge\left(J e_{k}\right\lrcorner e_{j}\right\lrcorner \psi^{-}\right) .
\end{aligned}
$$

So by (1)

$$
\left.\left.\left.\Lambda d\left(S_{\star} \psi^{-}\right)=-\delta S\right\lrcorner \psi^{+}+\left(\nabla_{e_{k}} S\right) e_{j} \wedge\left(e_{k}\right\lrcorner e_{j}\right\lrcorner \psi^{+}\right) .
$$


On the other hand, we have

$$
\begin{aligned}
\delta\left(S_{\star} \psi^{+}\right) & \left.\left.\left.\left.=e_{k}\right\lrcorner \nabla_{e_{k}}\left(S e_{j} \wedge e_{j}\right\lrcorner \psi^{+}\right) \stackrel{*}{=} e_{k}\right\lrcorner\left(\left(\nabla_{e_{k}} S\right) e_{j} \wedge e_{j}\right\lrcorner \psi^{+}\right) \\
& \left.\left.=-\delta S\lrcorner \psi^{+}-\left(\nabla_{e_{k}} S\right) e_{j} \wedge e_{k}\right\lrcorner e_{j}\right\lrcorner \psi^{+} \\
& \quad-(41) \\
\stackrel{(=1}{=} & -2 \delta S\lrcorner \psi^{+}-\Lambda d\left(S_{\star} \psi^{-}\right),
\end{aligned}
$$

thus proving (34). Let $\left.\left.\sigma:=\left(\nabla_{e_{k}} S\right) e_{j} \wedge\left(e_{k}\right\lrcorner e_{j}\right\lrcorner \psi^{+}\right)$denote the last summand in (41). A similar calculation easily shows that $J_{\star} \sigma=0$, so $\sigma$ belongs to $\Lambda^{(1,1)} M$. From (22) we thus get $\left.\alpha \Lambda d\left(S_{\star} \psi^{-}\right)=-\alpha(\delta S\lrcorner \psi^{+}\right)=-2 \delta S$. Finally, the relation (36) can be checked in the same way:

$$
\begin{aligned}
\Lambda \delta\left(S_{\star} \psi^{+}\right) & \left.\left.\left.=-\frac{1}{2} J e_{k}\right\lrcorner e_{k}\right\lrcorner\left(e_{j}\right\lrcorner \nabla_{e_{j}}\left(S_{\star} \psi^{+}\right)\right) \\
& \left.\left.\left.\left.\left.\left.=-\frac{1}{2}\left(e_{j}\right\lrcorner \nabla_{e_{j}}\left(J e_{k}\right\lrcorner e_{k}\right\lrcorner\left(S_{\star} \psi^{+}\right)\right)-e_{j}\right\lrcorner\left(\nabla_{e_{j}} J\right) e_{k}\right\lrcorner e_{k}\right\lrcorner\left(S_{\star} \psi^{+}\right)\right) \\
& \stackrel{*}{=} \delta \Lambda\left(S_{\star} \psi^{+}\right)=0 .
\end{aligned}
$$

\section{Concluding remarks}

So far we have identified the space of infinitesimal deformations of a given Gray structure with the space of coclosed primitive $(1,1)$-forms which are eigenforms of the Laplace operator for the eigenvalue 12. To proceed further there are two immediate options. One could try to compute the second derivative of a curve of Gray structures and obtain additional equations. However, this leads to quadratic expressions, which for the moment seem difficult to handle.

A second natural task is to consider the known homogeneous examples and to study the question of whether or not there exist at least infinitesimal deformations. This amounts to studying the Laplace operator on 2-forms on certain homogeneous spaces and should reduce to a tractable algebraic problem.

Since a nearly Kähler deformation always gives rise to an Einstein deformation, one could equally well ask for the existence of infinitesimal Einstein deformations on nearly Kähler manifolds. This issue was recently treated in [Moroianu and Semmelmann 2007].

The deformation problem for the standard nearly Kähler structure on $S^{6}$ has to be considered separately since the almost complex structure is no longer uniquely defined. However, since the round metric on $S^{6}$ has no Einstein deformations, the problem is much simpler in this case. Th. Friedrich [2006] showed that the action of the isometry group $\mathrm{SO}_{7}$ on the set of nearly Kähler structures on the round sphere $S^{6}$ is transitive. The isotropy group of this action at the standard nearly Kähler structure is easily seen to be the group $G_{2}$ (the stabilizer in $\mathrm{SO}_{7}$ of a vector cross product). The space of nearly Kähler structures on the round sphere is thus isomorphic to $\mathrm{SO}_{7} / G_{2} \simeq \mathbb{R P}^{7}$; see also [Butruille 2005, Prop. 7.2]. More geometrically, the set of nearly Kähler structures compatible with the round 
metric on $S^{6}$ can be identified with the set of nonzero real Killing spinors (modulo constant rescalings), and it is well known that the space of real Killing spinors on $S^{6}$ is isomorphic to $\mathbb{R}^{8}$.

The counterpart of Theorem 4.1 on $S^{6}$ can be stated as follows:

Theorem 5.1. Let $G:=\left(g, J, \omega, \psi^{+}, \psi^{-}\right)$be a Gray structure on $S^{6}$ such that $g$ is the round metric. Then the space of infinitesimal deformations of $G$ is isomorphic to the eigenspace for the eigenvalue 6 of the Laplace operator $\Delta$ on functions, and that space is, in particular, 7-dimensional.

Proof. Since there are no Einstein deformations on the round sphere, we may assume $\dot{g}=0$. From (14) we get $\varphi=0, h=0, S=0$, and in particular $\lambda=0$ too. Then Equation (38) gives $\xi=J d \mu$ and (39) shows that $\mu$ is an eigenfunction of $\Delta$ on $S^{6}$ corresponding to its first nonzero eigenvalue, 6 . These eigenfunctions (also called first spherical harmonics) satisfy $\nabla_{X} d \mu=-\mu X$ for all tangent vectors $X$. We define the infinitesimal $\mathrm{SU}_{3}$ deformation $\left.\dot{\omega}:=\xi\right\lrcorner \psi^{+}, \dot{\psi}^{+}:=-\xi \wedge \omega+\mu \psi^{-}$ and $\dot{\psi}^{-}:=-J \xi \wedge \omega-\mu \psi^{+}$. A short calculation easily shows that this indeed is a solution of the linearized system (25).

\section{References}

[Baum et al. 1991] H. Baum, Th. Friedrich, R. Grunewald, and I. Kath, Twistors and Killing spinors on Riemannian manifolds, Teubner-Texte zur Mathematik 124, B. G. Teubner, Stuttgart, 1991. MR 94a:53077

[Berger and Ebin 1969] M. Berger and D. Ebin, "Some decompositions of the space of symmetric tensors on a Riemannian manifold", J. Differential Geometry 3 (1969), 379-392. MR 42 \#993 Zbl 0194.53103

[Besse 1987] A. L. Besse, Einstein manifolds, vol. 10, Ergebnisse der Mathematik, Springer, Berlin, 1987. MR 88f:53087 Zbl 0613.53001

[Butruille 2005] J.-B. Butruille, "Classification des variétés approximativement kähleriennes homogènes”, Ann. Global Anal. Geom. 27:3 (2005), 201-225. MR 2006f:53060 Zbl 1079.53044

[Friedrich 2006] Th. Friedrich, "Nearly Kähler and nearly parallel $G_{2}$-structures on spheres", Arch. Math. (Brno) 42 (2006), 241-243. MR 2322410

[Gray 1976] A. Gray, "The structure of nearly Kähler manifolds", Math. Ann. 223:3 (1976), 233248. MR 54 \#6010 Zbl 0345.53019

[Moroianu and Semmelmann 2007] A. Moroianu and U. Semmelmann, "Infinitesimal Einstein Deformations of Nearly Kähler Metrics”, Preprint, 2007. arXiv math/0702455v1

[Nagy 2002] P.-A. Nagy, "Nearly Kähler geometry and Riemannian foliations", Asian J. Math. 6:3 (2002), 481-504. MR 2003m:53043 Zbl 1041.53021

[Obata 1962] M. Obata, "Certain conditions for a Riemannian manifold to be iosometric with a sphere”, J. Math. Soc. Japan 14 (1962), 333-340. MR 25 \#5479 Zbl 0115.39302

[Reyes-Carrión 1993] R. Reyes-Carrión, Some special geometries defined by Lie groups, $\mathrm{PhD}$ thesis, Oxford, 1993.

[Verbitsky 2007] M. Verbitsky, "Hodge theory on nearly Kähler manifolds”, Preprint, 2007. arXiv math/0510618v5 
[Wolf and Gray 1968] J. A. Wolf and A. Gray, "Homogeneous spaces defined by Lie group automorphisms. I”, J. Differential Geometry 2 (1968), 77-114. MR 38 \#4625a Zbl 0169.24103

Received June 7, 2007. Revised September 10, 2007.

ANDREI MOROIANU

Centre de Mathématiques

ÉCOLE POLYTECHNiQue

91128 Palaiseau CEDEX

FRANCE

am@math.polytechnique.fr

http://www.math.polytechnique.fr/cmat/moroianu/moroianu.html

PAUL-ANDi NAGY

DEPARTMENT OF MATHEMATICS

UNIVERSITY OF AUCKLAND

Private Bag 92019, Auckland Mail Centre

AUCKLAND 1142

NEW ZEALAND

nagy@maths.auckland.ac.nz

http://www.math.auckland.ac.nz/wiki/Paul_Andi_Nagy

UWE SEMMELMANN

MATHEMATISCHES INSTITUT

UNIVERSITÄT ZU KÖLN

WEYERTAL 86-90

D-50931 KÖLN

GERMANY

uwe.semmelmann@math.uni-koeln.de

http://www.mi.uni-koeln.de/ semmelma/ 